implemented smoke-free workplace laws designed to protect most workers. However, workers who work in other people's homes (such as home-care workers, tradespeople and nannies) are not protected by these laws, as smoking is not prohibited inside private homes. Previous research has suggested that domiciliary care workers, such as nurses making home visits to patients, are particularly heavily exposed to SHS. This research project sought to quantify that exposure in the context of wider occupational exposure to SHS.

Methods Through a programme of expert assessment, we developed a job exposure matrix (JEM) for SHS exposure among all classes of worker in the UK. Three raters assessed exposure to SHS for all UK occupations by 4-digit Standard Occupational Classification (SOC), rating likelihood, intensity and frequency of exposure.

To assess the extent of exposure to SHS among home-care workers, we conducted surveys of these workers in the NHS, two local authorities and a private organisation in Scotland. We conducted personal exposure monitoring with these groups of home-care workers, assessing their exposure to SHS by monitoring fine particulate matter $\left(\mathrm{PM}_{2.5}\right)$, air nicotine and changes in salivary cotinine over the course of a shift.

Results Our JEM indicated that around ten million workers in the UK may be occupationally exposed to SHS. Overall, 84 of 412 four-digit SOC codes (20.4\%) were considered likely to have at least $10 \%$ of workers experiencing some degree of non-incidental exposure to SHS during their duties. Exposure is estimated to be most severe among lower SES workers, particularly care workers. Our survey results indicated that many home-care workers are occupationally exposed to SHS. Local authority workers were more likely to be exposed than NHS workers, with $84 \%$ of council respondents reporting exposure during their work vs $15 \%$ of NHS respondents. Measurements revealed highly variable patterns of SHS exposure based on shift pattern and visit duration. Visits to smoking homes included peak $\mathrm{PM}_{2.5}$ concentrations in excess of $400 \mu \mathrm{g} / \mathrm{m}^{3}$, sixteen times the WHO guideline limit for $24 \mathrm{~h}$ periods.

Discussion SHS exposure remains a serious health concern for a considerable fraction of the UK working population. People in lower paid jobs are disproportionately affected by SHS at work, potentially a cause of health inequality. Home-care workers can experience frequent and high SHS exposure, and new policies are necessary to protect them from associated health harms.

\section{OP68 CAN SOCIETY AFFORD FURTHER DISINVESTMENT IN SMOKING CESSATION? A MICROSIMULATION STUDY TO QUANTIFY SMOKING CESSATION SERVICES IMPACTS IN ENGLAND}

Vincy Huang*, lain Buchan, Simon Capewell, Martin O'Flaherty, Chris Kypridemos. Public Health, Policy and Systems, University of Liverpool, Liverpool, UK

\subsection{6/jech-2021-SSMabstracts.68}

Background The UK smoking prevalence is decreasing, however, the inequality gap is increasing. A new UK Tobacco Strategy is being finalised and it urgently needs further underpinning research.Smoking cessation services (SCS) contributed around $15 \%$ of the reduction in UK smoking prevalence between 2001-2016. However, even these benefits are in jeopardy, given the proposed further funding cuts to SCS.
Using a previously validated microsimulation model, we quantitively compared three future SCS scenarios: maintaining, disinvesting, or enhancing services.

Methods We modelled the effectiveness and equity impacts of three scenarios over a 20-year time horizon:

A) a baseline of maintaining current SCS levels and trends;

B) assuming disinvestment (no SCS);

C) an enhanced SCS enabling 30\% of current smokers, aged between 30-79 years, to be supported in smoking cessation every five years. We used the validated IMPACT $_{\text {HINT }}$ microsimulation, an implementation of the IMPACT $_{N C D}$ framework, to estimate changes in smoking prevalence, disease burden, and economic impact. We simulated close-to-reality smoking histories, smoking-related diseases and lag times to disease. Population data were drawn from the Health Survey for England (HSE). We assumed the SCS one-year overall effectiveness of $8 \%$ quitting (reflecting published studies). We modelled the relapse probability post-cessation conditional on deprivation and years since cessation, informed by HSE. Standard UK Treasury discount rates were applied, and we report costs from a societal perspective, but no SCS costs included. We used R v4.04.

Results Preliminary results suggest that the disinvestment scenario could result in approximately 3000 (95\% Uncertainty Intervals: 990 to 5400) additional cases of cardiometabolic diseases, common cancers, and chronic obstructive pulmonary disease compared to the baseline scenario; most of them in the most deprived quintiles. The policy could result in about 4500 (2700 to 6700$)$ additional deaths and $£ 220 \mathrm{~m}(110 \mathrm{~m}$ to $380 \mathrm{~m})$ additional costs.In contrast, enhancing SCS could prevent or postpone approximately 1700 (420 to 3000) disease cases, most of them in the most deprived quintiles, and about 1700 (680 to 2700) fewer all-cause deaths. The policy could produce savings of $£ 270 \mathrm{~m}$ (120m to $460 \mathrm{~m}$ ) over the simulated period.

Conclusion Disinvesting in SCS is likely to be counterproductive, given their substantial health and economic benefits. Our model suggests that SCS provision needs to be continued at least at current levels. An enhanced service provision could be beneficial (after addressing issues of staff capacity and implementation costs).

\section{OP69 SOCIO-DEMOGRAPHIC DIFFERENCES IN SMOKING STATUS AND CESSATION BEFORE AND DURING EARLY PREGNANCY AMONG WOMEN IN ENGLAND: AN ANALYSIS OF THE NATIONAL MATERNITY SERVICES DATASET}

\begin{abstract}
1,2Danielle Schoenaker*, ${ }^{3}$ Judith Stephenson, 2,4,5Keith Godfrey, 2,4,5Mary Barker, ${ }^{1,2,6}$ Nisreen Alwan. 'School of Primary Care, Population Sciences and Medical Education, University of Southampton, Southampton, UK; ${ }^{2}$ NIHR Southampton Biomedical Research Centre, University of Southampton, University Hospital Southampton NHS Foundation Trust, Southampton, UK; ${ }^{3}$ Elizabeth Garrett Anderson Institute for Women's Health, University College London, London, UK; ${ }^{4}$ Human Development and Health, University of Southampton, Southampton, UK; ${ }^{5} M R C$ Lifecourse Epidemiology Unit, University of Southampton, Southampton, UK; ${ }^{6}$ NIHR Applied Research Collaboration Wessex, Southampton, UK
\end{abstract}

\subsection{6/jech-2021-SSMabstracts.69}

Background Smoking in pregnancy increases the risk of major adverse health outcomes for mothers and their offspring. The aim of this study was to describe socio-demographic differences in smoking before and during early pregnancy among women in England. 